\title{
Identifying Recurrent Laryngeal Nerve in Thyroid Surgery Using Relationship with Superior Parathyroid Gland and Tubercle of Zuckerkandl
}

\author{
Somesh Mozumder, ${ }^{1}$ Shirish Dubey, ${ }^{1}$ Aniruddha Dam, ${ }^{1}$ Anup Kumar Bhowmick ${ }^{1}$
}

\section{$\underline{\text { Introduction }}$}

\section{$\underline{\text { ABSTRACT }}$}

Recurrent laryngeal nerves (RLN) are particularly prone to injury during thyroid surgeries due to its intimate relationship and proximity with the gland. Zuckerkandl's tubercle (ZT) helps in preserving RLN intra operative.

\section{Materials and Methods}

A prospective study for identifying RLN in thyroid surgery using relationship with superior parathyroid gland and tubercle of Zuckerkandl was conducted on 50 thyroidectomy patients between August 2013 and February 2014.

$\underline{\text { Results }}$

In all cases ZT was identified. Temporary paralysis of RLN was seen in $3(6 \%)$ cases and permanent paralysis in $2(4 \%)$ of cases. Discussion

The site of greatest risk during thyroidectomy to the RLN is in the last $2-3 \mathrm{~cm}$ extralaryngeal course of the nerve. Relationship of recurrent laryngeal nerve with superior parathyroid gland and tubercle of Zukerkandl (ZT) is known.

Conclusion

Use of ZT and superior parathyroids as a landmark allows safe dissection of RLN.

Keywords

Thyroidectomy; recurrent laryngeal nerve; parathyroid

$\mathrm{Z}$ uckerkandl's tubercle (ZT) is defined as posterior extension of the lateral lobes composing of thyroid tissue only as per the name of Austrian anatomist Emil Zuckerkandl (1849-1910) who described it. ${ }^{1,2}$ Near this tubercle, on the posterior surface of thyroid gland superior parathyroid gland is located outside thyroid capsule and deep to pretracheal layer of deep cervical fascia. Below the above two structures, lies the recurrent laryngeal nerve approaching to enter the larynx behind cricothyroid joint and under the inferior constrictor muscle. Using these relationships, the recurrent laryngeal nerve can be safely and effectively identified and preserved while thyroidectomy surgeries.

\section{Material and Methods}

A prospective study for identifying recurrent laryngeal nerve in cases of thyroid surgery using relationship with superior parathyroid gland and tubercle of Zuckerkandl was conducted on 50 thyroidectomy patients between August 2013 and February 2014. Patients were evaluated clinically, by FNAC (Fine Needle Aspiration Cytology) and radiologically by ultrasonogram or neck computed tomography (CT) scans. Pre-operative vocal cord movement status were evaluated in every patient by indirect laryngoscopy. Total thyroidectomy and hemithyroidectomy (unilateral total lobectomy+isthmusectomy) are our procedures for the treatment of benign and neoplastic diseases of the thyroid. All operations were performed by a single

1 - Department of ENT and Head and Neck Oncology, Chittaranjan National Cancer Institute, Kolkata

Corresponding author:

Dr Somesh Mozumder

email: drsomeshmozumder@gmail.com 
surgeon in order to provide a standard dissection. Postoperative vocal cord movement status was evaluated in every patient by indirect-laryngoscopy immediately after surgery and every monthly for 6 months thereafter.

\section{Results and Analysis}

Out of total 50 patients who underwent thyroidectomy during the said period, $30(60 \%)$ underwent total thyroidectomy [FNAC proved malignancy], 15 underwent hemithyroidectomy [FNAC was suspicious of malignancy or proved to be benign] and 5 patients with extrathyroidal extension and or cervical nodal metastasis, underwent total thyroidectomy with central and lateral neck dissection on the diseased side.

Those who underwent simple hemithyroidectomy (15 patients) and only total thyroidectomy (30 patients), they neither had any extra-thyroidal extension nor neck node involvement; their thyroid anatomy was unaltered. So in their cases it was easy to identify and preserve recurrent laryngeal nerve using the relationship with tubercle of Zuckerkandl and superior parathyroid. Their post-operative vocal cord mobility and voice remained unaltered even after 6 months of follow up.

In the 5 cases $(10 \%)$ where there were gross extrathyroidal extension of disease and or the anatomy was altered or there was presence of central or lateral cervical nodal metastasis, the recurrent laryngeal nerve was difficult to locate during surgery as the reference land marks were already altered or lost. For them we tried extra capsular dissection as the superior parathyroid gland and recurrent laryngeal nerve lie outside the thyroid capsule.

Thus in 5 cases out of $50(10 \%)$ the tubercle of Zuckerkandl could not be identified.

3 patients, in whom ZT could not be identified, postoperatively revealed restricted mobility of vocal cord on the operated side with postoperative voice change but improved subsequently over 6 months as we followed up them monthly.

But 2 of them developed vocal cord palsy on one of the operated side with permanent voice change even after 6 months. (Table I)

\section{Discussion}

The recurrent laryngeal nerve supplies all muscles of larynx except cricothyroid and sensation of vocal cords and below. Injury to unilateral nerve causes ipsilateral vocal cord palsy in paramedian position and produces some degree of hoarseness which may recover in time due to compensation by the healthy cord which eventually crosses the midline to meet the paralysed one. Injury to both nerves is less likely to produce hoarseness as both the cord are in paramedian position but are prone to develop dyspnoea and stridor. Recurrent

Table I: Results of the Study

\begin{tabular}{|c|c|c|c|}
\hline \multicolumn{2}{|c}{$\begin{array}{c}\text { INCIDENCE } \\
\text { OF }\end{array}$ RECURRENT } \\
TYPE OF OPERATION DONE & NO. OF CASES & 0 & 0 \\
\hline $\begin{array}{c}\text { OnMPORARY } \\
\text { PERMANENT }\end{array}$ & 0 & 0 \\
\hline $\begin{array}{c}\text { Only total thyroidectomy (using said land } \\
\text { marks) }\end{array}$ & $30(60 \%)$ & 3 & 2 \\
\hline $\begin{array}{c}\text { Total thyroidectomy with central } \pm \text { lateral } \\
\text { neck dissection done (with altered local } \\
\text { anatomy) }\end{array}$ & $5(10 \%)$ & $3(6 \%)$ & $2(4 \%)$ \\
\hline \begin{tabular}{c} 
Total cases \\
\hline
\end{tabular}
\end{tabular}


laryngeal nerves are particularly prone to injury during thyroid surgeries due to its intimate relationship and proximity with the gland.

The left recurrent laryngeal nerve arises from the vagus in the mediastinum at the level of arch of aorta, loops around it and then ascends into the neck in the tracheo-oesophageal groove. Right nerve arises from the vagus at the level of subclavian artery, hooks around it and then ascends between the trachea and oesophagus.

Zuckerkandl's tubercle is a posterior extension of the lateral lobes composing of thyroid tissue. Near this tubercle, on the posterior surface of thyroid gland superior parathyroid gland is located outside thyroid capsule and deep to pretracheal layer of deep cervical fascia [more or less constant in location]. Below the above two structure lies the recurrent laryngeal nerve approaching to enter the larynx anterior to cricothyroid joint and under the inferior constrictor muscle.(Fig. 1).

At the apex of the Beahr's triangle (formed superiorly by inferior thyroid artery, below by recurrent laryngeal nerve and laterally by common carotid artery and apex is formed by meeting point of inferior thyroid artery and recurrent laryngeal nerve) the nerve is found below the Zuckerkandl's tubercle and superior parathyroid gland.

Otto Wilhelm Madelung had described in 1867 "posterior horn of the thyroid." 1,3 Emile Zuckerkandl has been reported in 1902 "processus posterior glandulae thyroideae." 1,2 The ZT is posterior extension of the gland

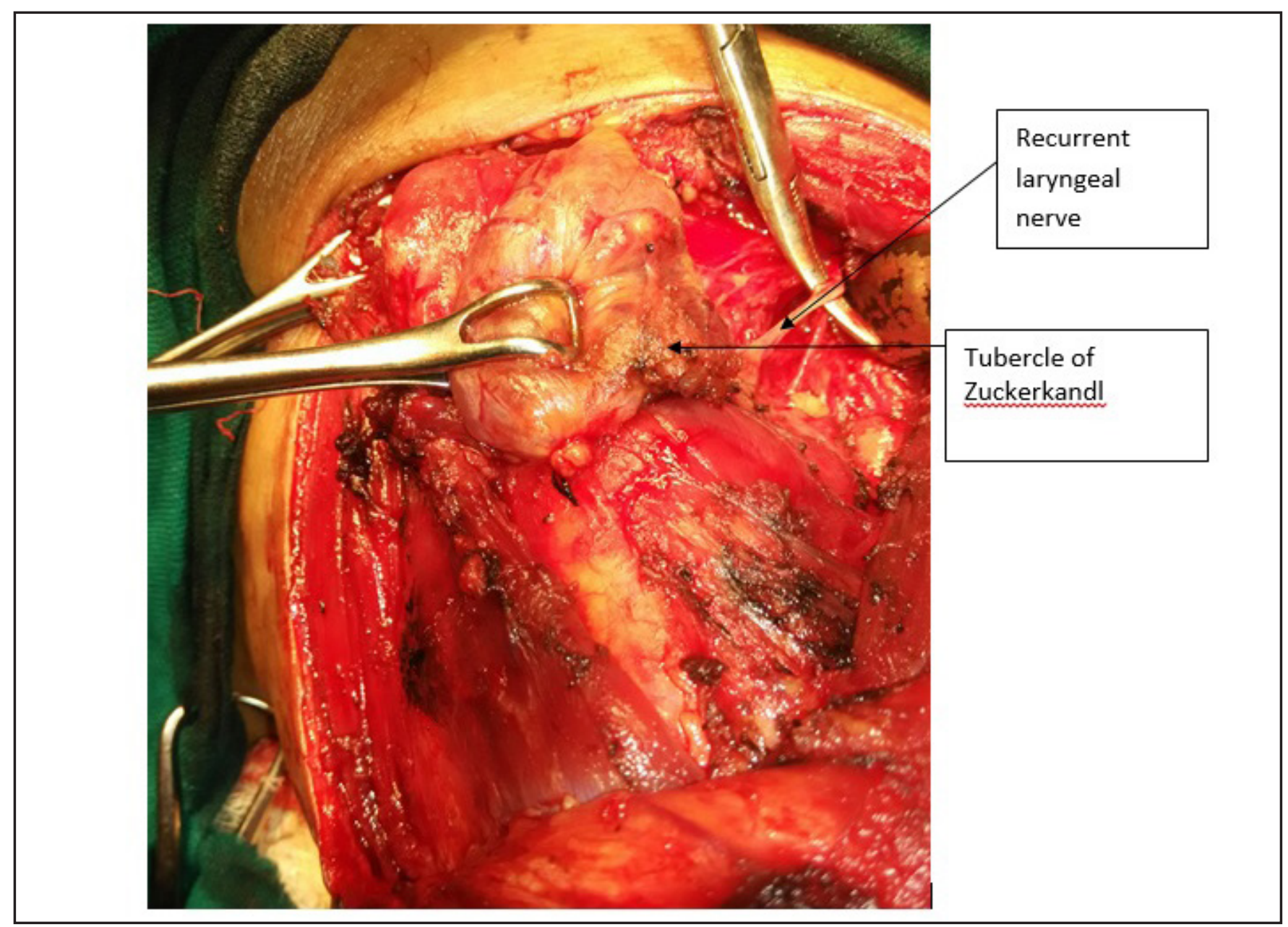

Fig. 1 Relation of recurrent laryngeal nerve with Tubercle of Zuckerkandl 
composed of thyroidal tissue. Surgical importance of the ZT arises from its relations with RLN. The resection of enlarged tubercle at posterior site of the thyroid requires delicate and careful dissection adjacent to the nerve. Identification of $\mathrm{ZT}$ and an understanding of the relationship between the ZT and RLN are essential for safety of thyroid operations. ${ }^{3,4,5}$ Surgeons generally perform thyroid operations on voluminous goitre that when present larger tubercles are observed on surgical specimens. Therefore, by surgical point of view an enlarged ZT parallel to goitre formation merits more interest than smaller one. It makes surgical dissection challenging at posterior site of the lateral lobes around RLN and inferior artery.

Numerous authors reported the incidence of ZT as more than $50 \%$ of their patients; Kaisha et al. ${ }^{6} 59 \%$, Hisham and Lukman ${ }^{4}$ 55\%, and Gauger el al. ${ }^{5} 63 \%$. On the other hand, Page et al. ${ }^{3}$ have identified ZT only in $7 \%$ of their patients.

RLN injury may be prevented by its full isolation based on intimate knowledge of the anatomy including all its variations. ${ }^{7}$ Some anatomical landmarks help surgeons identifying RLN.

$\mathrm{ZT}$ appears as an indicative arrow for the nerve and neurovascular crossing point in some patients. We can comment that after medial mobilization of the lobes, when present, ZT may be used as a landmark facilitating identification of the nerve. In our patient sample $90 \%$ of the patients had a recognisable ZT.

Many authors have previously stated that the ZT is a reliable and constant anatomical landmark as an arrow pointing the RLN. ${ }^{6,8,9-11}$ The site of greatest risk during thyroidectomy to the RLN is in the last $2-3 \mathrm{~cm}$ extralaryngeal course of the nerve before its laryngeal entry above the trunk of the inferior thyroid artery. ${ }^{12}$

Based on our findings ZT pointing, like an arrow head, neurovascular crossing point promotes surgeon's challenge to identify RLN. On the other hand, larger tubercle generally covers anterior surface of the nerve. Mobilization of the tubercle medially allows easy identification of the nerve at this dangerous site.

Relationship between the nerve and the tubercle leads to perform safer thyroid surgery.

The neighbouring of ZT and RLN is another important point for their relation. The resection of ZT for total thyroidectomy requires refined and meticulous dissection adjacent to the nerve. When enlarged by disease, the tubercle passes over the nerve like a bridge. This normal anatomical relationship is retained in the majority of cases. ${ }^{9}$

Excision of ZT is mandatory for completeness of thyroidectomy. Fine and delicate dissection with care around the $\mathrm{ZT}$ is also mandatory after identification and isolation of the RLN for preventing nerve injury.

Hisham and Lukman ${ }^{4}$ have previously reported that in $6 \%$ of dissection, the RLN was on the anterior surface of the tubercle. Gauger et al. ${ }^{5}$ have also reported that in $93 \%$ of patients with enlarged ZT, the RLN lays medial to the tubercle, and the nerve was found lateral to it in the remaining $7 \%$ of their cases. Anterior course of RLN is at highest risk of injury. In none of our patients, did we find the nerve to be on the anterior surface of the ZT.

The surgeon must be aware of the tubercle, and he must face the ZT without fear but with care. ${ }^{13}$ Identification of RLN is easier because of the constant relationship between these two structures at a level where the risk of injury is greatest. ${ }^{13}$ Identification of $\mathrm{ZT}$, an understanding of the relationship between the ZT and RLN, and isolation of the nerve before dissection of ZT are essential for performing safer thyroid surgery.

\section{Conclusion}

Zuckerkandl's tubercle which is defined as posterior extension of lateral lobes of the thyroid gland, is a common anatomical structure found in the majority of cases. Excision of the tubercle requires fine and meticulous dissection with great care because of close relationship between ZT and RLN.

Per operative identification of recurrent laryngeal using its relation with tubercle of Zuckerkandl and superior parathyroid in uncomplicated cases of hemi/ total thyroidectomy is a safe and easy method to avoid postoperative vocal cord paresis/palsy and voice change can be followed as a standard routine procedure. 


\section{References}

1. Mirilas P, Skandalakis JA. Zuckerkandl's tubercle: hannibal ad portas. Journal of the American College of Surgeons 2003;196:796-801

2. Shoja MM, Tubbs RS, Loukas M, Shokouhi G, Oakes WJ. Emil Zuckerkandl (1849-1910): anatomist and pathologist. Annals of Anatomy 2008;190:33-36

3. Yun JS, Lee YS, Jung JJ, Nam KH, Chung WY, Chang HS, et al. The Zuckerkandl's tubercle: a useful anatomical landmark for detecting both the recurrent laryngeal nerve and the superior parathyroid during thyroid surgery. Endocrine Journal 2008; 55(5):925-930.

4. Hisham AN, Lukman MR. Recurrent laryngeal nerve in thyroid surgery: a critical appraisal. ANZ Journal of Surgery 2002;72(12):887-889

5. Gauger PG, Delbridge LW, Thompson NW, Crummer P, Reeve TS. Incidence and importance of the tubercle of Zuckerkandl in thyroid surgery. European Journal of Surgery 2001;167(4):249254

6. Kaisha EW, Wobenjo A, Saidi H. Topography of the recurrent laryngeal nerve in relation to the thyroid artery, Zuckerkandl tubercle, and Berry ligament in Kenyans. Clinical Anatomy 2001;24:853-857
7. Gurleyik E. Three variations of the laryngeal nerve in the same patient: a case report. Journal of Medical Case Reports 2001;5:266

8. Page C, Cuvelier P, Biet A, Boute P, Laude M, Strunski V. Thyroid tubercle of Zuckerkandl: anatomical and surgical experience from 79 thyroidectomies. Journal of Laryngology and Otology 2009;123(7):768-771

9. Pelizzo MR, Toniato A, Gemo G. Zuckerkandl's tuberculum: an arrow pointing to the recurrent laryngeal nerve (constant anatomical landmark). Journal of the American College of Surgeons 1998;187(3):333-336

10. Yalçin B, Poyrazoğlu Y, Ozan H. Relationship between Zuckerkandl's tubercle and the inferior laryngeal nerve including the laryngeal branches. Surgery Today 2007; 37(2):109-113

11. Yalçin B, Ozan H. Relationship between the Zuckerkandl's tubercle and entrance point of the inferior laryngeal nerve. Clinical Anatomy 2007;20(6):640-643

12. Serpell JW. New operative surgical concept of two fascial layers enveloping the recurrent laryngeal nerve. Annals of Surgical Oncology 2010;17(6):1628-1636

13. Toniato A, Boschin IM. The Zuckerkandl tubercle. American Journal of Surgery 2008;195(2):277 